\title{
An Intelligent Tutoring System with Variable Levels of Instructional Support for Instructing Natural Deduc- tion
}

\author{
Kazuhisa Miwa \\ Hitoshi Terai \\ Nana Kanzaki \\ Ryuichi Nakaike
}

Graduate School of Information Science, Nagoya University

miwalis.nagoya-u.ac.jp, http://miwalab.cog.human.nagoya-u.ac.jp/

(affiliation as previous author)

terailis.nagoya-u.ac.jp

Junior College, Nagoya Woman's University

kanzaki@cog.human.nagoya-u.ac.jp

Graduate School of Education, Kyoto University

keywords: Intelligent tutoring; Production system; Instructional help; Natural deduction; Client-server framework

\begin{abstract}
Summary
We present an intelligent tutoring system that teaches natural deduction to undergraduate students. An expert problem solver in the system provides basic instructional help, such as suggesting the use of a rule in the next step of solving a problem and indicating the inference drawn by applying the rule. The system provides help by using a complete problem solver as an expert instructor. Students learning with our tutoring system can vary the degree of help they receive (from low to high and vice versa). Empirical evaluation showed that the system enhanced the problem-solving performance of participants during the learning phase, and these performance gains were carried over to the post-test phase. The analysis of participants' interactions with the system revealed the between-participants adaptation of students, meaning that participants with lower scores learned using higher levels of assistance than those with higher scores. In addition, the analysis revealed the within-participants adaptation of students, meaning that they adaptively changed levels of support according to their learning progress and the degree of difficulty of the problem.
\end{abstract}

\section{Introduction}

This paper presents an intelligent tutoring system that teaches natural deduction (ND) to undergraduate students. ND is a proof calculus in which logical reasoning is expressed by inference rules that are closely related to natural ways of reasoning [Barwise 03]. Studentes (as users) of our tutoring system learn inference rules and strategies for applying these rules, such as strategies for inferring the proposition $\neg Q \rightarrow \neg P$ from the premise $P \rightarrow Q$. Following is an example solution process:
(1) $P \rightarrow Q \quad$ Premised
(2) $\neg Q \quad$ Premised
(3) $P \quad$ Premised
(4) $P \rightarrow Q \quad$ Reiteration of (1)
(5) $Q \quad$ Condi. Elim. from (3) and (4)
(6) $Q \quad$ Reiteration of (2)
(7) $\neg P \quad$ Neg. Intro. from (3), (5), and (6)
(8) $\neg Q \rightarrow \neg P \quad$ Condi. Intro. from (2) and (7)

Several universities include ND in their curricula for teaching the basics of logical thinking and formal reasoning. Traditionally, ND has been taught using paper and pencil. The instructor explains the functions of the inference rules and presents example problems, and students learn the inference rules by solving the problems. However, using ND to solve problems is relatively complex because it involves selecting a single appropriate rule from various candidate rules, applying the rule to the appropriate propositions, and inferring the results. Students often have difficulties determining the correct rules to use, and may stop learning because they do not know how to proceed. In these situations, students require help from the instructor. However, in traditional classroom settings, which may have more than 100 students, individual tutoring is sometimes impossible.

To overcome the abovementioned problems, we developed an intelligent tutoring system. Our system provides basic instructional help, such as suggesting the use of a rule in the next step of solving a problem and indicating 
the inference drawn by applying the rule. The system provides help by using a complete problem solver as an expert instructor. The problem solver performs ND using its technical knowledge and inference mechanisms. Our tutoring system is an expert problem solver, but it possesses neither the knowledge nor the strategies required for providing instructions. The expert solver solves problems and instructs students on what to do next on the basis of its own attempts to solve the problems. However, the expert solver does not know how to help students learn effectively, i.e., it does not know when, how, or to what degree it should offer help.

Hint presentation is an essential factor in intelligent tutoring systems [Mercier 08, Roll 06, Roll 07b, Roll 07a, Roll 11]. In our system, students are responsible for the instructional management. Although our system has no management functions for controlling the degree of help students receive, students learning with our tutoring system can vary the degree of help they receive (from low to high and vice versa). This is a critical functionality for intelligent tutoring systems. Typically, expert systems are used for improving and maximizing the performance of problem solving in a specific domain. When an expert system is used in conjunction with human users, the system provides users with as much information as possible to achieve the highest possible performance of problem solving. However, when we use expert systems for tutoring, we should consider another important goal, i.e., learning [Dweck 86]. During the learning phase, students using such systems should stress their learning rather than achieving the maximum problem solving performance. This requires the ability to control the degree of help students receive when solving problems.

The issue of the degree of help students should receive when solving problems is termed the assistance dilemma. Koedinger \& Aleven (2007) raised a crucial question: "In order to achieve optimal learning, what balance should learning environments strike between giving and withholding assistance?" [Koedinger 07]The dilemma is that in certain cases, a high level of assistance improves learning, but in others, it elicits superficial responses that do not require thinking on the part of students [Koedinger 08, Miwa 12]. Therefore, even though students may successfully solve example problems using the tutoring system, they may not generate high scores in the post-test phase, because superficial learning prevents them from learning the essentials.

In this paper, we first present a detailed description of the functionality of our system. Next, we evaluate our system from two viewpoints. We examine if the direct indications provided by our system improve not only the problem solving performance of students in the learning phase, but also their learning gains in the post-test phase. Additionally, we analyze whether students solve example problems for learning by adaptively controlling the degree of help they receive by using the "level of support" (LOS) function of our system.

\section{Tutoring System}

\section{$2 \cdot 1$ Client-server framework}

Our system consists of an expert solver implemented as a rule-based expert system, and tutor terminals that are used by students to interface with the system. The system was implemented on a client-server framework. Miwa and his colleagues designed the system using their webbased production system architecture, termed "DoCoPro" [Miwa 13]. The expert solver is implemented on the server and provides the complex ND inferences. Client computers connected to the server are used as tutor terminals and perform simple interface processing. The results of tutoring sessions are saved as log data on the server. Because our system uses a client-server framework, it can operate in any educational environment where various types of computers are used, e.g., high performance, low performance, and computers with different operating systems.

\section{$2 \cdot 2 \quad$ Rule-based expert system}

The database of our system does not contain a set of ND problems and their solutions. Rather, the expert solver solves each problem on demand. Our system is designed on the basis of a production model consisting of a working memory whose layout is consistent with the structure of ND problems, and production rules corresponding to the inference rules and strategies for solving the ND problems. Our system covers 14 representative inference rules and 10 strategies.

Figure 1 provides a summary of how the expert solver and tutor terminal work. Consider the following deduction status:

$$
\begin{array}{lll} 
& P & \text { Premised } \\
& Q & \text { Inferred by Condi. Elimi. } \\
& \text { Target proposition }
\end{array}
$$

To obtain the target proposition $P \rightarrow Q$, first $P$ was premised, and then $Q$ was inferred (in this example, $Q$ was inferred by applying Condition Elimination). At this point, the inference rule "Condition Introduction" is ready to fire. The Condition Introduction rule is expressed as follows: 


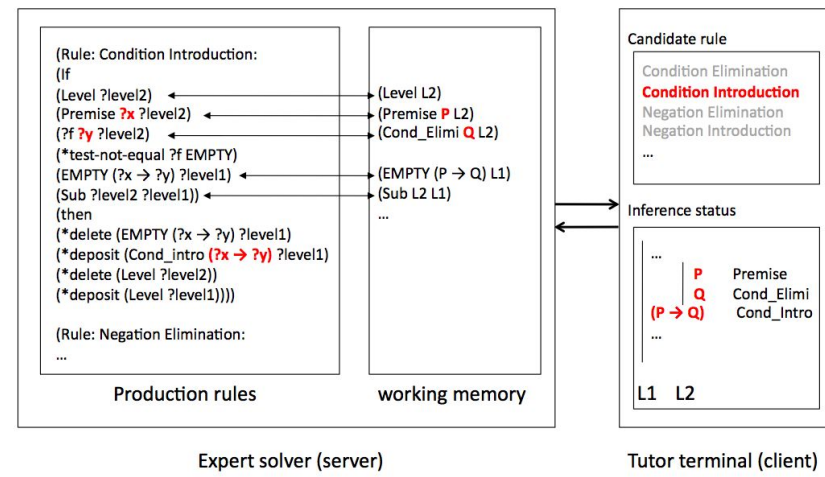

Fig. 1 Help generation mechanism of production system. Because the rule Condition Introduction is ready to fire, it is recommend for inference. Next, the rule is applied to propositions $P$ and $Q$ because they bind variables $? x$ and $? y$ in the if-clause of the rule. Finally, the rule deposits proposition $P \rightarrow Q$ in working memory, and we obtain the inferred result $P \rightarrow Q$.

If

$P$ is premised in the subproof

$Q$ is obtained in the proof level

Then

$$
P \rightarrow Q \text { is drawn into the upperproof }
$$

Figure 1 illustrates how the production system generates help indications. The working memory contains the deduction status presented above. L1 is an upperproof level of a subproof level L2. Because the if-clause of the rule Condition Introduction matches the contents of the working memory, help indications are drawn. First, the expert solver informs the tutor terminal that the rule Condition Introduction could be applied because it is ready to fire. Second, variables $? x$ and $? y$ in the if-clause of the rule are bound by propositions $P$ and $Q$, respectively, which are contained in the working memory. Therefore, the expert solver announces that the rule should be applied to propositions $P$ and $Q$. Finally, we obtain the inferred result $P \rightarrow Q$, because the rule deposits this proposition in the working memory.

\section{$2 \cdot 3$ Levels of support management}

Figure 2 shows an example screenshot of the tutor terminal. Students are presented with lists of inference rules and strategies. The system automatically runs the rule or strategy selected by the user. Then, it presents either the complete inference result or a template in which the result is partially blanked. The system helps students select the appropriate rule and strategy. In Figure 2, the leftside window shows the current status of deduction. The middle window shows the candidate inference rules and strategies. The upper-right window shows the LOS selector that enables students change the degree of help. Using

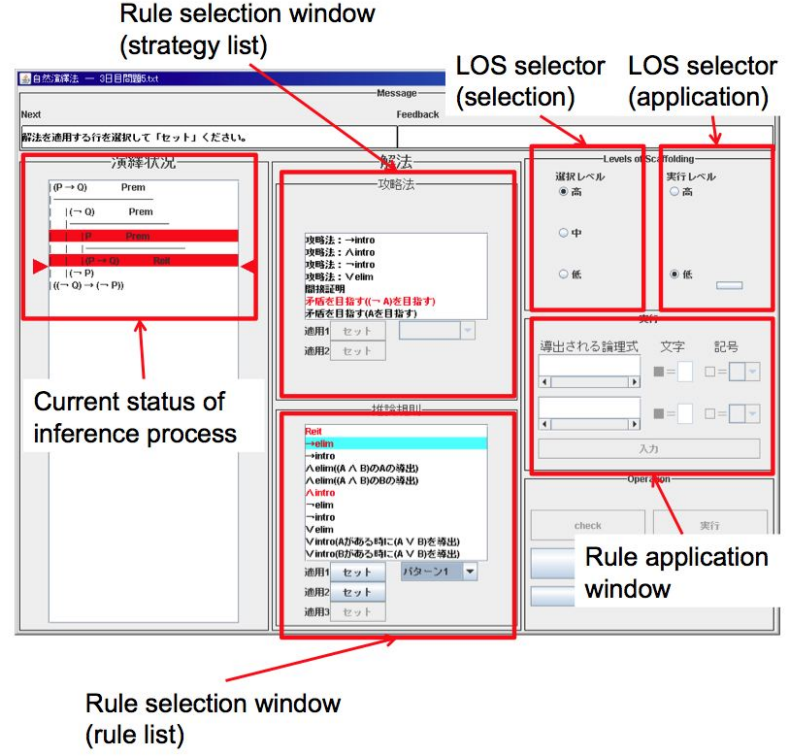

Fig. 2 Example screenshot of the tutor terminal.

the lower-right window, students input propositions drawn from the process of deduction.

Students can control the degree of help they receive using the LOS selector. Students select the desired LOS with respect to two viewpoints: rule selection and rule application.

LOS for rule selection:

- Level 3 (high): The system presents applicable candidates (rules and strategies) and the propositions to which the rules should be applied. For example, in the middle window in Figure 2, the system proposes that three highlighted inference rules and one strategy could be applied. When " $\rightarrow$ elim" is selected, $P$ and $P \rightarrow Q$ are highlighted in the left window, indicating that the selected rule should be applied to these propositions.

- Level 2 (middle): The system presents only applicable candidates (rules and strategies). When this level is selected, students are required to find the propositions to which the selected rule should be applied, without receiving support from the system.

- Level 1 (low): The system presents only a set of inference rules and strategies (no support is provided).

LOS for rule application:

- Level 2 (high): The system infers a proposition and automatically presents it in the left window. Shortly after students select an inference rule and the propositions to which the rule will be applied, the system displays the current status of deduction.

- Level 1 (low): The system infers a proposition, but presents only partial information of the inferred re- 


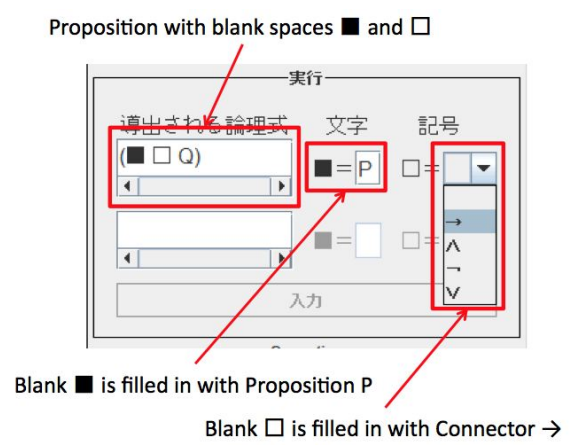

Fig. 3 Example input window used in the Level 1 LOS to apply rules.

sult. Students are required to complete the inference process by filling terms in blank spaces. Figure 3 shows an example input screen where the user has filled in the proposition $P \rightarrow Q$ using the template provided by the system.

Our principle for setting up the LOS levels was that we maximized the hint range from low to high between the maximum hint (i. e., the bottom-out hint: see [Aleven 00]) and the minimum hint (i. e., no hint). In rule selection, Level 1 corresponds to the no hint situation; but Level 3 does not reach the bottom-out hint level because our problem solver cannot identify one single rule that determines what to do next completely, but just present multiple candidate rules for application. Additionally, in rule application, Level 3 functions as the bottom-out hint level, but Level 1 does not correspond to the no hint situation because the template used in the interface may give students hint information for rule application.

\section{$2 \cdot 4$ System verification}

Next, we verified that our system can successfully solve all 35 problems contained in a representative textbook [Todayama 00] used for teaching ND in Japan. Table 1 lists the inference rules and strategies used to solve the 35 problems.

\section{Evaluation}

To evaluate the usefulness of our system, we designed and conducted an experiment that addressed the following two research questions. First, we examined whether learning with our tutoring system is an improvement over learning with the traditional method using paper and pencil. Second, we examined how the LOS management function of our tutoring system was used by students. For this examination, we evaluated the LOS management behavior of students with respect to between-participants and withinparticipants adaptation. Between-participants adaptation occurs when students with greater abilities select a lower LOS while learning and students with lesser abilities select a higher LOS. Within-participants adaptation occurs when students adaptively change the LOS according to their progress in learning and the degree of difficulty of the problem.

\section{$3 \cdot 1$ Procedure}

A total of 49 students participated in the experiment. Of these, 33 were assigned to the experimental group and the remaining 16 to the control group. We assigned twice as many participants to the experimental group because this group would later be divided into two subgroups on the bases of their post-test scores. Participants were assigned to one of the two groups randomly. This assignment strategy for the experiment is adequate enough because all participants were involved in the identical school of a university, and prior to the practice, no participants were familiar with the learning contents (i. e., natural deduction).

During the initial phase of the experiment, the participants in both groups learned the basics of ND using handout materials and viewing an instructional video. Next, during the 80-min learning phase, the participants in the experimental group learned ND by solving six example problems using our tutoring system. Concurrently, the participants in the control group learned ND by solving the same six problems using only handout materials; the handout materials contained all the information required to solve the problems. In both groups, the participants were informed that after the practice, they solve two test problems for an examination in which they are required to mark higher scores. In the learning phase, participants solved six problems, two of which were difficult and required a second-order subproof, two were easy and either did not require a subproof or required only a first-order subproof.

After the learning phase, the participants of both groups solved two post-test problems printed on test sheets. One was an easy problem that required a first-order subproof and the other was a difficult problem that required a secondorder subproof.

\section{$3 \cdot 2$ Post-test scores}

We analyzed the post-test scores in order to answer our first research question. In the learning phase, the participants in the experimental group solved an average of 5.21 example problems, while those in the control group solved only an average of 2.69 problems. Statistical analysis shows that the difference between the two groups is significant $(t(47)=5.71, p<0.01)$, which means that dur- 
Table 1 Lists of rules and strategies applied to solve the 35 problems contained in an ND textbook (Todayama, 2000).

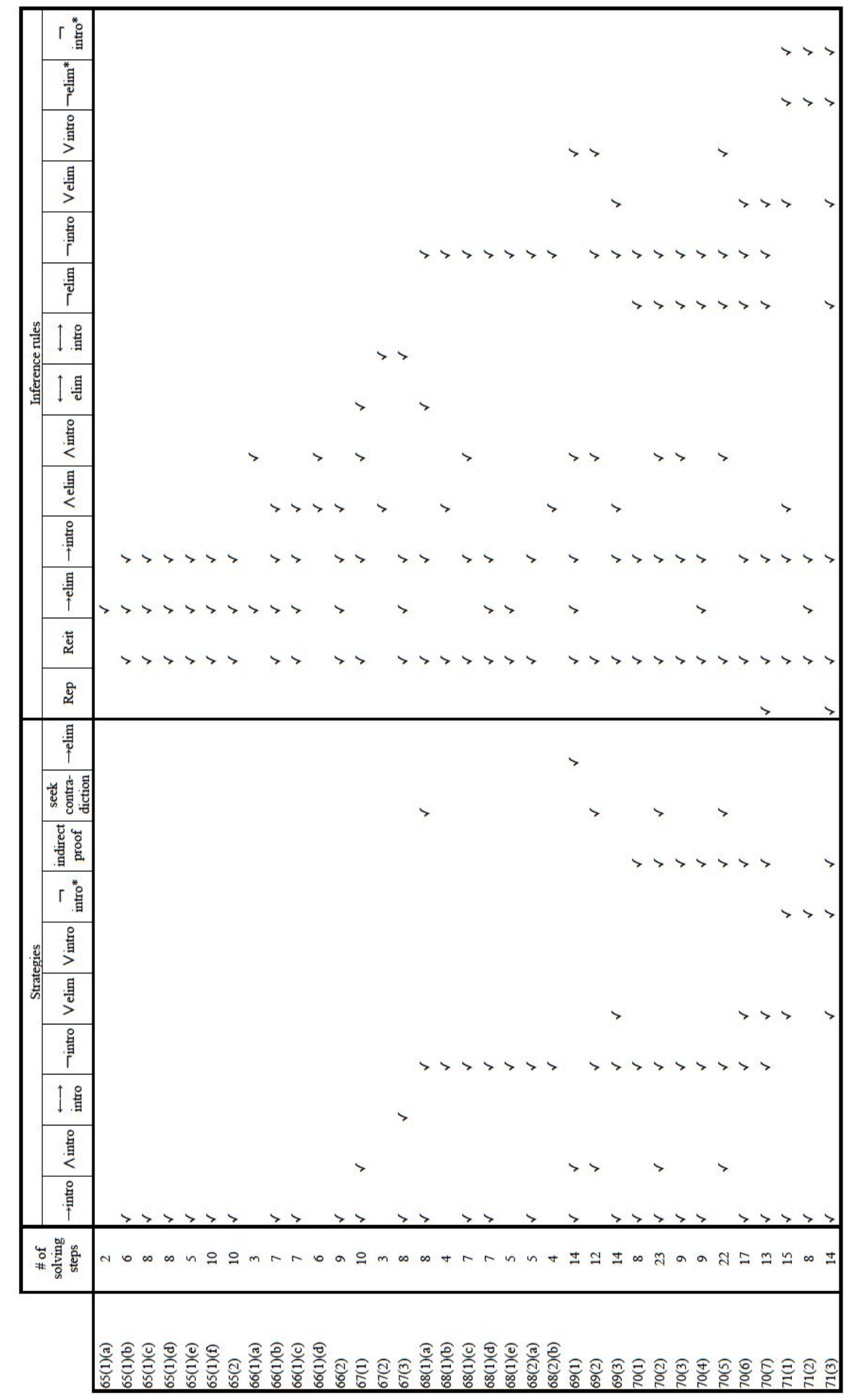



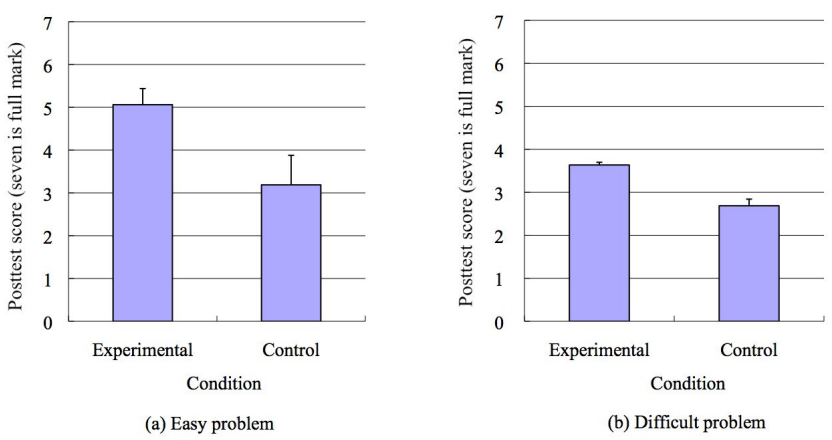

Fig. 4 Average post-test scores in experimental and control conditions.

ing the learning phase, the tutoring system successfully enhanced the problem-solving performances of the participants. A critical question is whether this performance gain was carried over to the post-test scores, where the tutoring system was not used.

Figure 4 shows the post-test scores. Statistical analysis shows that the average score for solving the easy problem was significantly higher in the experimental group than in the control group $(t(47)=2.59, p<0.05)$. Conversely, the average score for solving the difficult problem was not higher statistically in the experimental group than in the control group $(t(47)=1.42$, n.s.). This result indicates that participants who learned using our tutoring system performed better only when solving the easy problem.

\section{$3 \cdot 3$ LOS management}

Next, we analyzed the LOS management activities of the participants in the experimental group. In solving each of the six problems, the average ratio of participants who did not vary a LOS at all was 0.180 . All participants manipulate a LOS at least once throughout solving the six problems. First, to evaluate whether between-participants adaptation occurred, we divided the participants into two groups on the basis of their average scores for the two post-test problems, and formed a lower- and a higher-score group. As a result, 18 participants were assigned to the higher-score group and 15 were to the lower-score group. Our hypothesis was that the participants in the lower-score group learned using higher levels of assistance than those in the higher-score group.

Figure 5 shows the result of this analysis. Statistical analysis shows that for rule selection, the average LOS of the higher-score group was significantly lower than that of the lower-score group $(t(31)=2.19, p<0.05)$, which supports our hypothesis. However, for rule application, there was no difference in the LOS averages $(t(31)=1.24$, n.s.).

To evaluate the within-participants adaptation of stu-
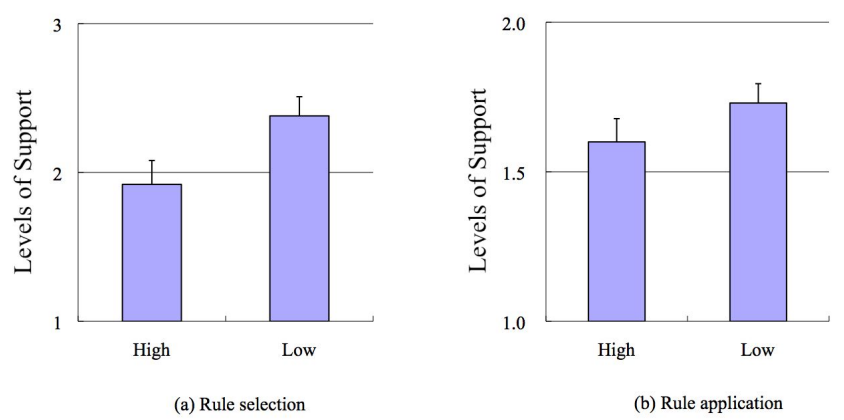

Fig. 5 Levels of assistance versus post-test scores.

dents, we examined two types of behavior: self-fading and adaptation with respect to problem difficulty. First, we examined the well-known behavior for adapting LOS, called self-fading [Atkinson 03], where students lower the LOS by themselves. In this experiment, participants were allowed to solve problems at their own pace. Some participants quit solving a problem, moved to other problems, and then revisited the initial problem and attempted to complete it. In certain cases, participants attempted to solve a problem more than two times. We compared the average LOS of the first attempt with that of the second and following attempts. When two or more attempts were performed, we used the average score of the second and following attempts. In terms of the second and following attempts, 117 cases in rule selection and 116 cases in rule application were recorded. The former was divided into 80 cases in the higher-scored group and 37 cases in the lower-scored group; the latter was divided into 80 cases in the higher-scored group and 36 cases in the lower-scored group. The small number in the lower-scored group is because the participants in the group often faced impasses in the learning phase and solved only limited number of example problems. Our hypothesis was that participants in the first attempt used a higher LOS than in subsequent attempts.

Figure 6 shows the results of our analysis. The ratio of irregular cases in which a LOS in the second and following attempts was higher than a LOS in the first attempt was 0.085 for rule selection and 0.259 for rule application. For rule selection, statistical analysis shows that the average LOS in the second and following attempts was significantly lower than that in the first attempt $(t(116)=6.10$, $p<0.01$ ), which supports our hypothesis. The ratio of irregular cases in which a LOS in the second and following attempts was higher than a LOS in the first attempt was 0.085 . However, for rule application, there was no difference between the first and subsequent attempts $(t(115)<$ 1, n.s.). 

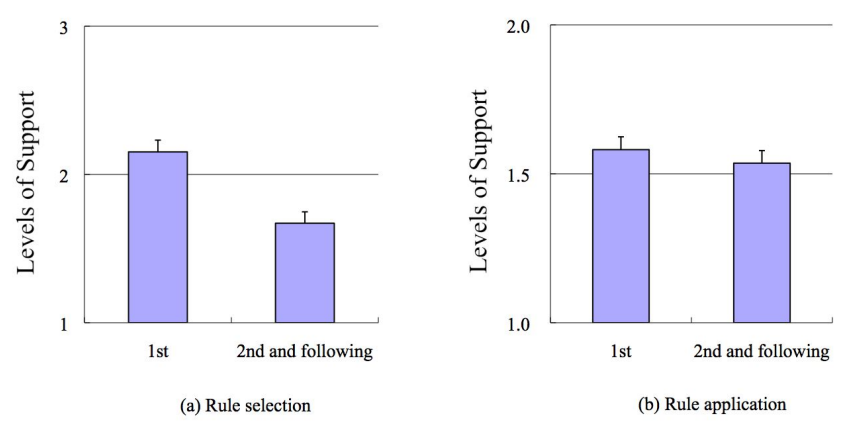

Fig. 6 Levels of assistance versus number of attempts.
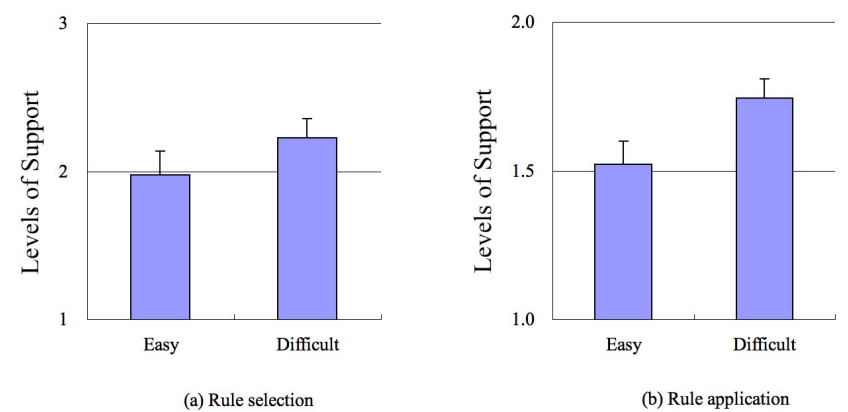

Fig. 7 Levels of assistance versus problem difficulty.

Moreover, we expected that participants would adapt the LOS according to the degree of difficulty of the problem. Two of the six problems (neither easy nor difficult problems) were excluded from analysis because it was impossible to define the difficulty of problems based on their syntactic structures (see the definition of easy and difficult problems in 3.1). Our hypothesis was that participants solving difficult problems would select a higher LOS than when solving easy problems.

Figure 7 shows the results. The ratio of irregular participants whose LOSs were higher when solving easy problems than when solving difficult problems was 0.219 for rule selection and 0.156 for rule application. For rule selection, statistical analysis shows that when participants solved difficult problems, the average LOS was significantly higher than when solving easy problems $(t(31)=$ 2.59, $p<0.05)$. Similarly, for rule application, when participants solved difficult problems, the average LOS was significantly higher than when solving easy problems $(t(31)=4.58, p<0.01)$. The results of the analyses of self-fading and adaptation with respect to problem difficulty support our hypothesis that within-participants adaptation occurred.

\section{Discussion and conclusions}

In terms of the usefulness of our tutoring system, our results show that during the learning phase, participants in the control group solved only less than half the problems. This means that participants faced serious difficulties during this phase. In contrast, participants in the experimental group solved almost all problems, which means that the system enabled the participants to overcome the difficulties. The benefits of learning with the tutoring system were carried over in the post-test phase only when solving the easy problem. No statistical improvement in the performance was observed when solving the difficult problem. This indicates a limitation of the current system. Basically a way of tutoring of the system is relatively simple; it offers students only candidate rules determining what to do next, and removes impasses for problem solving. However, the system does not instruct conceptually any declarative knowledge that may lead students to deeper understandings about the functions of inference rules and strategies.

This may indicate a limitation in the direct approach used by our system to provide instructions, such as simply indicating the next step to be performed. In the future, we will address this limitation.

Our evaluation also concerned the usability of the LOS management function of our system. Although many preceding studies have indicated that learners behave irrationally in seeking help [Wood 99, Aleven 00, Aleven 04], our overall results indicate that participants who used our tutoring system adaptively managed the LOS. Participants with greater abilities learned using a lower LOS compared to those with lesser abilities, which supports our hypothesis that between-participants adaptation occurred. Participants lowered the LOS as their learning progressed from the first to subsequent attempts. Moreover, they raised the LOS when solving difficult problems, which supports our hypothesis that within-participants adaptation occurred. As above, we confirmed that students properly manage the help degree for their learning, but did not examine to what degree such adaptive behavior contributes their learning gains. To understand such a causal relation, we need a controlled experiment in which participants learn in either a low or high LOS condition. More empirical investigations of natures of help seeking behavior based on such experiments are an important future direction for our further investigation (see a preliminary trial in [Miwa 12]).

Generally, students very often fell in trouble when selecting adequate rules and strategies. Therefore, a function of the tutoring system presenting candidate rules for rule 
selection is crucial. The support for rule application is relatively easily established by a simple schema-based inference execution mechanism. However, the support for rule selection is more complex. It needs an exhaustive search for matching inference statuses in the working memory and conditions for applying each inference rule in the production memory. The most crucial part of our learning system is the mechanisms for such a rule selection support embodied on the production system architecture. Other tutoring systems exist for teaching ND. The tutoring system called "Fitch" provides students with templates for reasoning, and automatically performs reasoning [Barwise 03]. However, this system does not provide information about which rule should be applied at each phase of reasoning. As mentioned above, instructional assistance in rule selection is more important to introductory students than in rule application, and hence, this is a critical limitation of "Fitch."

Croy et al. (2007) developed an intelligent tutoring system for teaching how to construct propositional proofs [Croy 07, Stamper 11]. To identify the best option for a specific stage of reasoning, they drew on previously acquired log data from reasoning processes of other students, and used a Markov decision model to infer the next step in reasoning. Consequently, they implemented a "Hint Factory" and successfully improved the learning of introductory students in deductive logic courses. Because of the limited inference abilities of our system, while providing help, it sometimes narrows the possible rules or strategies to a few candidates rather than a single one. Compared to our tutor, the tutor of Croy et al. always provides the best candidate rule for the next reasoning step. However, our system can handle problems that have not been solved in advance. Conversely, the tutoring system of Croy et al. must accumulate training data for the Markov model, which is time consuming. They have tried to shorten the preliminary trials [Stamper 10]. The most important feature of our system is that it solves given problems on demand. To use this advantage, we have begun to expand our system for "Learning through problem posing," where in a group setting each student solves original problems generated by other members with help of our tutoring system.

\section{$\diamond$ References $\diamond$}

[Aleven 00] Aleven, V. and Koedinger, K. R.: Limitations of Student Control: Do Student Know when they need help?, in Proceedings of the 5th International Conference on Intelligent Tutoring Systems, ITS 2000, pp. 292-303 (2000)

[Aleven 04] Aleven, V., McLaren, B., Roll, I., and Koedinger, K. R.: Toward Tutoring Help Seeking: Applying Cognitive Modeling to Meta-cognitive skills, in Proceedings of the 7th International Confer- ence on Intelligent Tutoring Systems, ITS 2004, pp. 227-239 (2004)

[Atkinson 03] Atkinson, R. K., Renkl, A., and Merrill, M. M.: Transitioning From Studying Examples to Solving Problems: Effects of Self-Explanation Prompts and Fading Worked-Out Steps, Journal of Educational Psychology, Vol. 95, pp. 774-783 (2003)

[Barwise 03] Barwise, J. and Etchemendy, J.: Language, Proof and Logic, CSLI publications (2003)

[Croy 07] Croy, M., Barnes, T., and Stamper, J.: Towards an Intelligent Tutoring System for propositional proof construction, in Proceedings of European Computing and Philosophy Conference, pp. 145-155 (2007)

[Dweck 86] Dweck, C. S.: Motivational Processes Affecting Learning, American Psychologist, Vol. 41, pp. 1040-1048 (1986)

[Koedinger 07] Koedinger, K. R. and Aleven, V.: Exploring the Assistance Dilemma in Experiments with Cognitive Tutors, Educational Psychology Review, Vol. 19, pp. 239-264 (2007)

[Koedinger 08] Koedinger, K. R., Pavlik, P., Mclaren, B., and Aleven, V.: Is it Better to Give than to Receive? The Assistance Dilemma as a Fundamental Unsolved Problems in the Cognitive Science of Learning and Instruction, in Proceedings of the 30th Annual Conference of the Cognitive Science Society, pp. 2155-2160 (2008)

[Mercier 08] Mercier, J. and Frederiksen, C.: The structure of the help-seeking process in collaboratively using a computer coach in problem-based learning, Computers \& Education, Vol. 51, pp. 17-33 (2008)

[Miwa 12] Miwa, K., Terai, H., and Nakaike, R.: Tradeoff between Problem-solving and Learning Goals: Two Experiments for Demonstrating Assistance Dilemma., in Proceedings of the 34th Annual Conference of the Cognitive Science Society, pp. 2008-2013 (2012)

[Miwa 13] Miwa, K., Morita, J., Nakaike, R., and Terai, H.: Learning through Intermediate Problems in Creating Cognitive Models., Interactive Learning Environments. (in press). (2013)

[Roll 06] Roll, I., Aleven, V., McLaren, B., Ryu, E., Baker, R., and Koedinger, K. R.: The Help Tutor: Does Metacognitive Feedback Improve Students' Help-Seeking Actions, Skills, and Learning?, in Proceedings of the 8th International Conference on Intelligent Tutoring Systems, ITS 2006, pp. 360-369 (2006)

[Roll 07a] Roll, I., Aleven, V., McLaren, B., and Koedinger, K. R.: Can Help Seeking Be Tutored? Searching for the Secret Sauce of Metacognitive Tutoring, in Proceedings of the 13th International Conference on Artificial Intelligence in Education, AIED 2007, pp. 203-210 (2007)

[Roll 07b] Roll, I., Aleven, V., McLaren, B., and Koedinger, K. R.: Designing for metacognition - applying cognitive tutor principles to the tutoring of help seeking, Metacognitiion Learning, Vol. 2, pp. 125-140 (2007)

[Roll 11] Roll, I., Aleven, V., McLaren, B., and Koedinger, K. R.: Improving students' help-seeking skills using metacognitive feedback in an intelligent tutoring system, Learning and Instruction, Vol. 21, pp. 267-280 (2011)

[Stamper 10] Stamper, J. C., Barnes, T., and Croy, M.: Enhancing the Automatic Generation of Hints with Expert Seeding, in Proceedings of the 10th International Conference on Intelligent Tutoring Systems, ITS 2010, pp. 31-40 (2010)

[Stamper 11] Stamper, J., Eagle, M., Barnes, T., and Croy, M.: Experimental Evauation of Automatic Hint Generation for a Logic Tutor, in Proceedings of the 15th International Conference of Artificial Intelligence in Education, AIED 2011, pp. 345-352 (2011)

[Todayama 00] Todayama, K.: Learning Logic through Building it, Nagoya University Publisher (2000)

[Wood 99] Wood, H. and Wood, D.: Help seeking, learning and contingent tutoring, Computers and Education, Vol. 33, pp. 153-169 (1999)

〔担当委員 : KOJIRI, Tomoko〕

Received May 15, 2013. 


\section{Author's Profile}

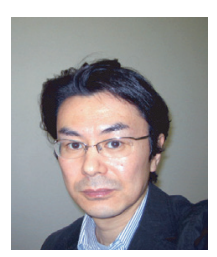

\section{MIWA, Kazuhisa (Member)}

KAZUHISA MIWA is a professor in Nagoya University. He is investigating human higher order cognition such as creativity based on the model based approach and the human experimentation method.

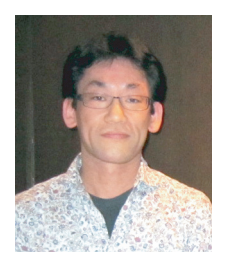

\section{TERAI, Hitoshi (Member)}

HITOSHI TERAI is an designated associate professor in Nagoya University / CREST-JST(Japan Science and Technology). His research topics focus on cognitive science studies including problem solving, information seeking behavior, and human system interaction.

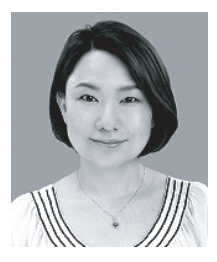

\section{KANZAKI, Nana}

KANZAKI NANA is an assistant professor in Junior College, Nagoya Woman's University. Her research topics focus on cognitive psychological studies of diagrammatic representation, and its implications to educational technology.

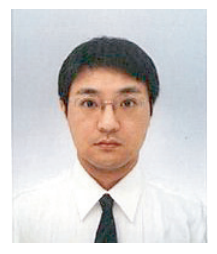

\section{NAKAIKE, Ryuichi (Member)}

RYUICHI NAKAIKE is an assistant professor in Kyoto University. His research interests include educational technology, learning support system, and human system interaction. 